\title{
Numerical Simulation of a Three-Dimensional Fish-like Body Swimming with Finlets
}

\author{
Shizhao Wang* , Xing Zhang and Guowei He \\ LNM, Institute of Mechanics, Chinese Academy of Sciences, Beijing 100190, \\ P.R. China.
}

Received 15 May 2010; Accepted (in revised version) 15 May 2011

Available online 30 November 2011

\begin{abstract}
The swimming of a 3D fish-like body with finlets is numerically investigated at $\operatorname{Re}=1000$ (the Reynolds number is based on the uniform upstream flow and the length of the fish-like body). The finlets are simply modeled as thin rigid rectangular plates that undulate with the body. The wake structures and the flow around the caudal peduncle are studied. The finlets redirect the local flow across the caudal peduncle but the vortical structures in the wake are almost not affected by the finlets. Improvement of hydrodynamic performance has not been found in the simulation based on this simple model. The present numerical result is in agreement with that of the work of Nauen and Lauder [J. Exp. Biol., 204 (2001), pp. 2251-2263] and partially supports the hypothesis of Webb [Bull. Fish. Res. Bd. Can., 190 (1975), pp. 1-159].
\end{abstract}

AMS subject classifications: 76D05, 76Z10, 76D17

Key words: Finlets, fish swimming, Immersed Boundary method, wake structure.

\section{Introduction}

The high performance of fish locomotion has attracted the attention of both biologists and engineers for a long time. The hydrodynamic efficiency and maneuverability have been the subject of numerous studies. For example, Bainbridge [1] studied the relationship of body length, tail beat frequency and swimming speed; $\mathrm{Wu}[2]$ used the two-dimensional undulating plate theory to predict the swimming performance; and Lighthill [3] extended the three-dimensional slender-body theory to calculate the hydrodynamics. Recently, Zhu et al. [4] studied the three-dimensional flow structures and vorticity control using a fish-like model and nonlinear inviscid flow method. Zhang et al. [5] investigated experimentally the interaction of a deformable filament (a passively swimming fish) with the surrounding flows. Later, Zhu and Peskin [6] performed a numerical simulation based on

${ }^{*}$ Corresponding author. Email addresses: wangsz@lnm.imech.ac.cn (S. Z. Wang), zhangx@lnm.imech.ac.cn (X. Zhang), hgw@lnm.imech.ac.cn (G. W. He) 
the experiment of Zhang et al. [5]. Lauder et al. [7] analyzed the pectoral fin swimming in sunfish experimentally. Dong et al. [8] quantitatively characterized the propulsive performance of the pectoral fin using numerical simulation. A recent review on fish swimming was given by Bandyopadhyay [9]. Compared with experimental study, numerical simulation has some advantages in control of fish-body kinematics and availability of data for the entire flow field. Thus it is playing a more and more important role in studying fish swimming, especially the role of appendages, such as dorsal fin, anal fin and pelvic fin etc. In this paper, the role of finlets is investigated using numerical simulations.

Finlets are small non-retractable fins located on the body margins between the dorsal/anal fins and the caudal fins [10]. The fish with finlets usually is capable of performing high-performance locomotion, such as mackerel, tuna and bonito. Some of the finlets are rigid and flat, while others are flexible. The total surface area of the finlets can reach $15 \%$ of the caudal fin.

Because of the special position and the relatively large area proportion of the finlets, it is suspected that the finlets play a role in fish locomotion. Walters [11] proposed that the finlets direct flow longitudinally along the body and prevent rolling; while Webb [12] and Lindsey [13] proposed that the motion of the finlets can reduce the drag in swimming by preventing the separation of the boundary layer. Nauen and Lauder [14] studied the flow around the caudal peduncle and finlets of the chub mackerel scomber japonics, and claimed that the finlets have a hydrodynamic effect on local flow. Bandyopadhyay [9] postulated that the finlets enhance the main thrust jet by producing a pair of counterrotating vortices with an intervening jet converging toward the tail. To summarize, the role of finlets is still controversial.

In order to investigate the role of finlets, the present work numerically simulates the swimming of a three-dimensional fish-like body with and without finlets. The finlets are modeled as continuous thin rigid plates attached to the contraction region of the dorsal and ventral ridges. The finlets undergo undulation with the body. With this finlets model, the results of the present work indicate that the finlets direct the local flow around the caudal peduncle, but have little effect on wake structures. This result partially supports the hypothesis of Webb [12] and Lindsey [13], and is in agreement with the work of Nauen and Lauder [14]. However, the finlets model used in the present work is different from the morphology of a real fish. To further verify this hypothesis, simulation based on the geometries and kinematics of real fish will be performed in the future work.

This paper is organized as follows. The numerical model and method are given in

Section 2. The numerical observations and discussions are presented in Section 3. Finally, conclusions are drawn in Section 4.

\section{Numerical model and method}

\subsection{Numerical model}

In the present work, we consider a fish-like body undergoing prescribed undulation in a uniform flow. The unsteady three-dimensional incompressible Navier-Stokes equations 
are solved to simulate the swimming of the fish-like body. The governing equations are

$$
\begin{aligned}
& \nabla \cdot \mathbf{u}=0, \\
& \frac{\partial \mathbf{u}}{\partial t}+\mathbf{u} \cdot \nabla \mathbf{u}=-\nabla p+\frac{1}{R e} \nabla^{2} \mathbf{u}+\mathbf{f},
\end{aligned}
$$

where $\mathbf{u}$ and $p$ are the velocity and pressure respectively. $\mathbf{f}$ is the body force representing the boundary in the Immersed Boundary (IB) method. $R e=U L / v$ is the Reynolds number, where $U$ is the velocity of the uniform upstream flow; $L$ the length of the fish-like body and $v$ the kinematic viscosity.

A three-dimensional fish-like body representing the shape of a tuna is employed in the simulation. The parameters of the body shape used in this study are taken from the work of Zhu et al. [4], with a little modification around the caudal peduncle according to Wu and Wang [15]. The geometry of the fish-like body is shown in Fig. 1.

The undulation of the fish-like body is prescribed in a body-fixed coordinate system $x-y-z$. The motion is in the form of a traveling backbone wave with smoothly varying

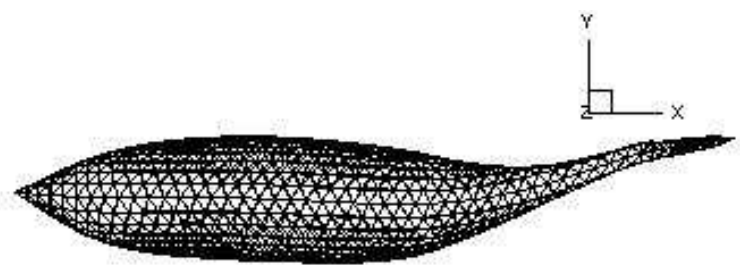

(a)

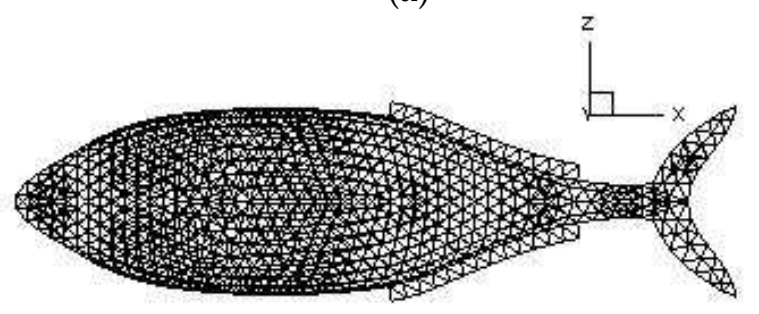

(b)

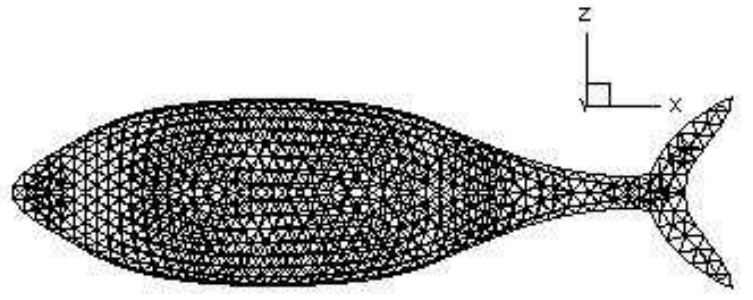

(c)

Figure 1: Geometry of the fish-like body. (a) top view, at the time when the caudal peduncle is at the maximum undulation position; (b) side view of the body with finlets; (c) side view of the body without finlets. 
amplitude and phase speed. The following form of the backbone wave is used in the present work [4],

$$
\begin{aligned}
& y(x, t)=a(x) \sin \left(k_{w} x-\omega t\right), \\
& a(x)=c_{1} x+c_{2} x^{2} .
\end{aligned}
$$

Here $k_{w}=2 \pi / \lambda$ is the wave number, where $\lambda$ is the wavelength. $\omega$ is the circular frequency. $x=0$ is located at a distance of 0.3 body length from the nose and the positive $x$-axis is in the direction from the nose to the tail. $c_{1}$ and $c_{2}$ are two parameters that represent the variation of amplitude along $x$.

The caudal fins undergo a pitch motion about the caudal peduncle. The frequency of the pitch motion is the same as that of the backbone wave-like motion; while its phase angle lags that of the lateral motion of the caudal peduncle by a constant. The angle of attack of the caudal fin, $\theta$, is given by

$$
\theta=\alpha \sin \left(k_{w} x_{p}-\omega t-\varphi\right) .
$$

Here $\alpha$ is the maximum angle of attack and $x_{p}$ is the $x$-coordinate of the peduncle; $\varphi$ the phase difference between the motion of the peduncle and that of the caudal fin.

\subsection{Numerical method and settings}

The Immersed Boundary (IB) method is used to simulate the swimming of the fish-like body. In this method, the effect of the complex geometries on the external flows is representing as a body-force term, thus simplify the mesh-generation procedure for complex geometries. The IB method is first induced by Peskin [16] to study a deformable boundary interacting with a fluid. This method was later extended to treat rigid boundary and now many variants of IB method exist [17-19]. The Navier-Stokes equations (2.1) and (2.2) are solved on a Cartesian grid. The basic solver uses the discrete stream function approach. This approach has the advantages that the divergence-free condition is satisfied to machine precision and there are no splitting errors associated with it. The discrete form of the Navier-Stokes equations can be expressed by a matrix form as:

$$
\left[\begin{array}{cc}
\mathbf{R A} & \mathbf{R G} \\
-\mathbf{D} & \mathbf{0}
\end{array}\right]\left[\begin{array}{c}
\mathbf{C} s^{n+1} \\
q
\end{array}\right]=\left[\begin{array}{c}
\mathbf{R} r^{n} \\
0
\end{array}\right]-\left[\begin{array}{c}
\mathbf{R} q_{b} \\
0
\end{array}\right]+\left[\begin{array}{c}
\mathbf{R} f \\
0
\end{array}\right]
$$

where $s, q$ and $f$ are the discrete stream-function, pressure, and body force respectively. $q_{b}$ is the boundary condition on pressure. A, G, D, R, and $\mathbf{C}$, are the implicit operator, gradient operator, divergence operator, rotation operator and the curl operator respectively. An unstructured, triangular mesh is used to track the surface of the fish-like body. The direct forcing IB method is used to enforce the non-slip boundary condition on the complex surface of the fish. For the technical details of this numerical methodology and some benchmark validations, please refer to [20]. 
The present simulation is conducted in a domain of $6 L \times 1 L \times 1 L$. The non-slip boundary condition is applied on the surface of the moving boundary. The boundary condition at the inlet is a uniform flow. The outflow boundary condition is used at the outlet. Symmetric boundary conditions are set at the front, back, top and bottom boundaries.

The parameters to prescribe the motion of the fish-like body are: $\lambda=1.22, \omega=2 \pi$, $c_{1}=0.0, c_{2}=1.55, \alpha=\pi / 6.0, \varphi=\pi / 2.0$, where $\lambda$ is non-dimensionalized by the length of the body $L$, and $\omega$ is non-dimensionalized by the characteristic circular frequency $U /(2 \pi L)$ [15].

The Reynolds number is taken as 1000. A uniform mesh of $300 \times 50 \times 50$, with mesh size $h=0.02 L$, is used in the simulation. The number of Lagrangian nodes representing the surface of the fish-like body is 1200 . The time step is dynamically computed by fixing the maximum CFL number at 0.5 in the simulation. The maximum CFL number is defined as

$$
C F L_{\max }=\max \left(\frac{U_{i}\left(h_{1}+h_{2}\right) d t}{h_{1} h_{2}}\right), \quad i=1,2,3, \cdots, \text { number of face, }
$$

where, $U_{i}$ is the normal component of velocity on face $i ; h_{1}$ and $h_{2}$ the distances between the face $i$ and the centroids of its two neighboring cells.

To investigate the influence of computational domain and mesh size on the numerical results, the simulation of the fish-like body swimming with finlets is conducted on two more cases: one case is set for a larger computational domain of size $12 L \times 2 L \times 2 L$ with the same mesh size $h=0.02 \mathrm{~L}$ and another is set for the same computational domain of size $6 L \times 1 L \times 1 L$ with a finer mesh size $h=0.01 L$. The results of the different cases are shown in Fig. 2, where $C_{x}=F_{x} /\left(0.5 \rho U^{2} L^{2}\right)$ is the streamwise force in fish-like body swimming and $C_{y}=F_{y} /\left(0.5 \rho U^{2} L^{2}\right)$ is the lateral force. The force differences in the three cases are within $5 \%$. The differences in flow structures of the three cases are also limited. The contours

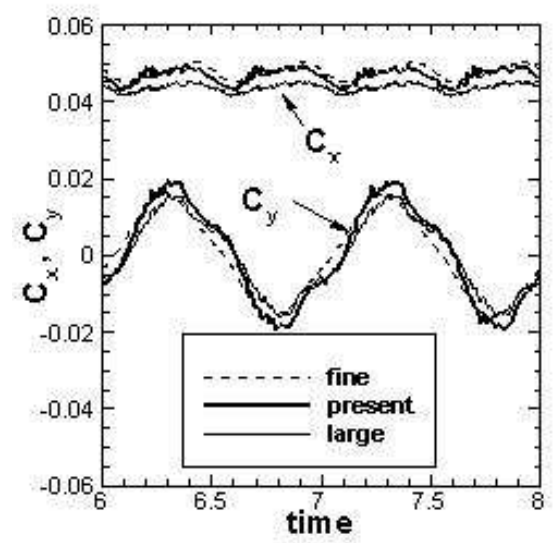

Figure 2: The streamwise and lateral forces on the fish-like body at different computational domains and mesh size. The dashed-lines represent the results on a finer mesh. The thin solid lines show the results in a larger computational domain. The thick solid lines are the results used in present work. 


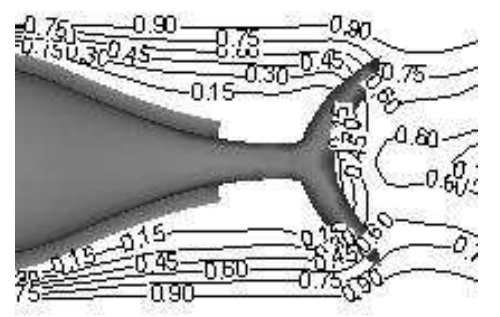

(a)

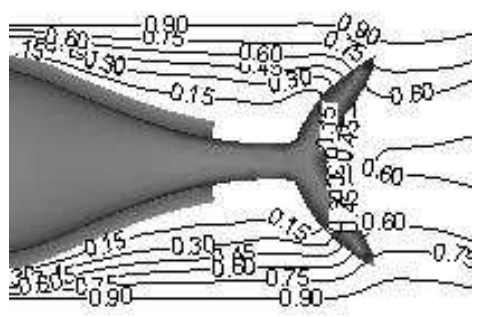

(b)

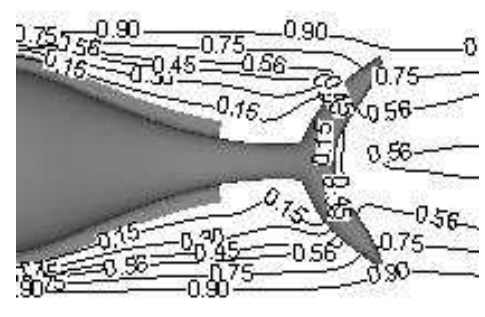

(c)

Figure 3: The contours of streamwise velocity in plane $y=0$ at the same time (acceleration phase). The results come from three different cases: (a) the grid size and computational domain used in present simulations; (b) the larger computational domain; (c) the finer grid size.

of streamwise velocity in plane $y=0$ at the same time (acceleration phase) are shown in Fig. 3.

\section{Numerical observations and discussions}

\subsection{Wake structures}

The wake structures behind three-dimensional flapping bodies have been studied extensively. The wake structures can be categorized as two types: single-row wake and double-row wake. The appearance of the wake structure depends on the Reynolds number, aspect ratio and kinematics of flapping motion. The wake structures of the swimming fish-like body in this work are found to be of single-row type and very similar to these of a mackerel-like fish in the work of Borzjani and Sotiropoulos [21]. The wake structures of the swimming fish-like body with finlets are shown in Fig. 4. The wake structures are identified using the Q-criterion and colored with the values of streamwise vorticity. The wake structures behind the fish-like body show the characteristics of a single-row type: the flow structures consist of a series of hairpin-like vortices. These vortices braid together and are located within a narrow strip region along the horizontal axis of the fish body. The topology of the flow structures is also very similar to that of a flapping ellipsoidal foil in the work of Dong et al. [22]. It is worth pointing out that the evolution of braided vortices does not clearly exhibit the phenomena of "spanwise narrowing". This is different from the result of a flapping ellipsoidal foil in the work of Dong et al. [22], but in agreement with that of Ellenrieder et al. [23]. The appearance of the spanwise narrowing seems to be dependent on the morphology and the kinematics of the flapping foil.

The wake structures of the swimming fish-like body without finlets are almost the same as that with finlets. Fig. 5 shows the comparison of the flow structure near the caudal peduncle. The structures in the red circle of Fig. 5(a) are generated by the motion of finlets. A detailed examination of these vortices shows that they are the streamwise vor- 


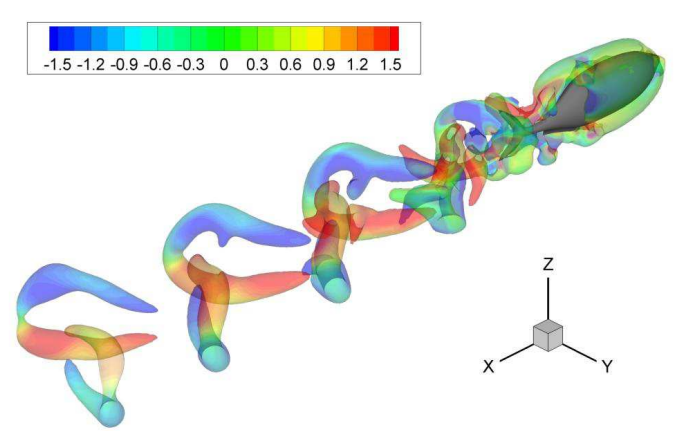

Figure 4: Wake structures for the swimming of fish-like body with finlets. The wake structures are identified using the $\mathrm{Q}$-criterion $(\mathrm{Q}=2.0)$. The wake structures are colored with the values of streamwise vorticity. The flow structures consist of a series of hairpin-like vortices. These vortices braid together and are located within a narrow strip region along the horizontal axis of the fish body.

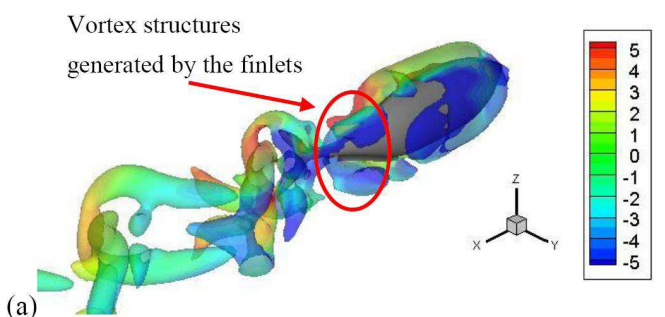

(b)

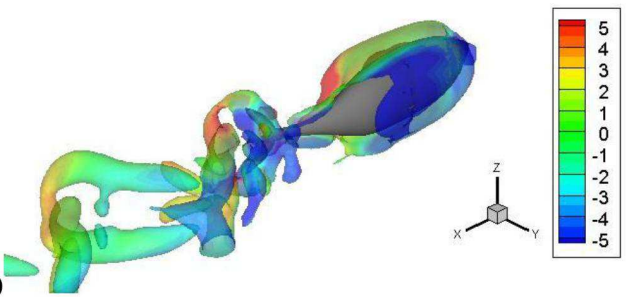

Figure 5: Comparison of the flow structures of a fish-like body swimming with and without finlets; (a) with finlets; (b) without finlets. The wake structures are identified using the $Q$-criterion $(Q=2.0)$. The wake structures are colored with the values of crosswise vorticity ( $z$ direction in the figure). The vortex structures in the red circle in (a) are the structures generated by the finlets. The flow structures outside the caudal peduncle region are almost the same.

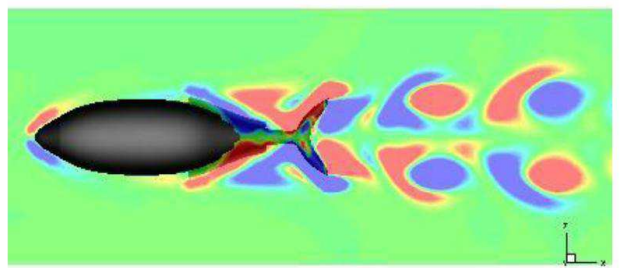

(a)

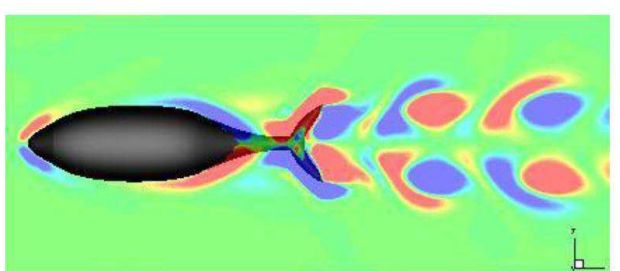

(b)

Figure 6: Comparison of the flow structures of a fish-like body swimming with and without finlets; (a) with finlets; (b) without finlets. The contours of streamwise vorticity on $y=0$ plane are shown. There is only a slight difference near the region of the caudal peduncle, whereas the structures outside this region are not affected. 
ticities generated by the lateral motion of the peduncle. Both the crosswise vorticity and spanwise vorticity in this region can induce a compression to these streamwise vortical structures and reduce their strength significantly. This effect of vorticity attenuation occurs immediately downstream of the finlets, this is the reason why the finlets have little effect on the far wake structures. This can be shown clearly in Fig. 6, where the contours of streamwise vorticity at the $y=0$ plane are plotted. There is only a little difference between the two near the region of the caudal peduncle, and the flow structures outside this region are almost not affected at all.

Because of the almost identical characteristics in wake structures, the forces on the fish-like body with and without finlets are only slightly different. No improvement in the performance has been found with the addition of finlets under the present conditions. Indeed there is a little increase in drag. This is a support of the drag-increasing hypothesis presumed by Nauen and Lauder [14], though the morphology and the kinematics of the present finlets model are different from the real fish.

\subsection{Flow around the caudal peduncle}

To investigate the effect of finlets on local flow, streamlines and streamwise vorticity around the caudal peduncle are examined. Fig. 7 shows the projection of streamlines in $x$-z plane $(y=0)$ for the fish-like body with and without finlets respectively. Fig. 7(a) and (b) show the streamlines at the accelerating phase of stroke. The direction of the longitudinal velocity is modified only a little by the motion of finlets.

A slice parallel to the $y-z$ plane at $x=0.6$ is extracted to investigate the flow across the peduncle. The slice is just after the finlets for the fish-like body with finlets. The streamlines and streamwise vorticity contours are shown in Fig. 8. Both flows at the accelerating and decelerating phase of stroke are plotted. The streamlines are more asymmetric and the streamwise vorticity is larger for the fish-like body with finlets. These results indicate that the finlets redirect the flow across the peduncle and enhance the three-dimensional

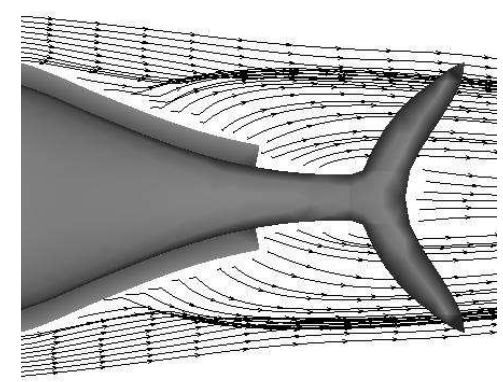

(a)

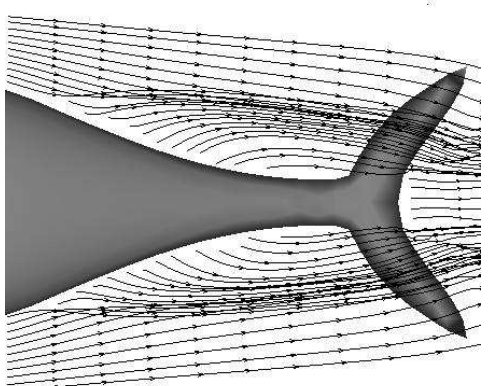

(b)

Figure 7: Projection of streamlines in $x-z$ plane $(y=0)$ for the fish-like body with and without finlets $(a)$ with finlets; (b) without finlets. 


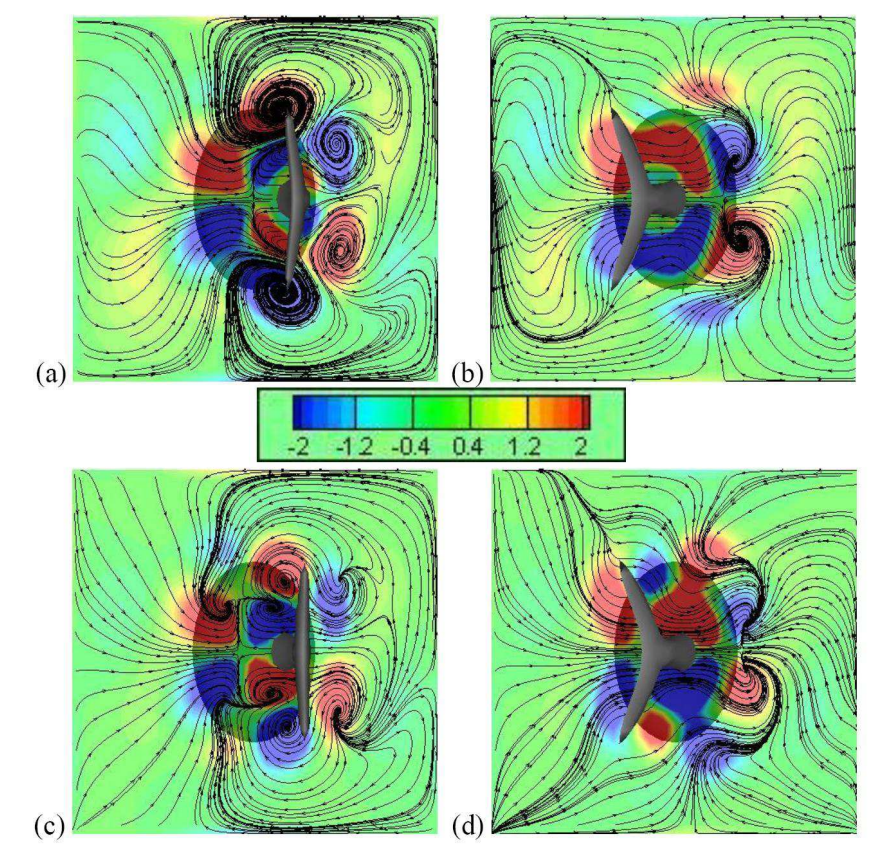

Figure 8: Flows in $y-z$ plane at $x=0.6$ for the accelerating and decelerating phase of a stroke. The in-plane streamlines are plotted. The direction of viewing is from tail to head. The contours are colored with the values of streamwise vorticity. (a) decelerating phase of stroke for fish-like body with finlets; (b) accelerating phase of stroke for fish-like body with finlets; (c) decelerating phase of stroke for fish-like body without finlets; (d) accelerating phase of stroke for fish-like body without finlets.

effect of the flow. The present result partly supports the hypothesis of Webb [12] and Lindsey [13]. That is, finlets deflect flow across the caudal peduncle, and have little effect on the longitudinal flow. The result is also in agreement with the observation of Nauen and Lauder [14]. To verify the entire hypothesis of Webb [12] and Lindsey [13], a simulation at higher Re number and with the real geometry and kinematics may be needed.

\section{Conclusions}

The swimming of a three-dimensional fish-like body with and without finlets is numerically simulated. The fish-like body undergoes the prescribed undulation in a uniform flow. A simplified finlets model is used in the simulation and the effect of the finlets is investigated. Improvement of hydrodynamic performance has not been found under the present conditions. It is found that the finlets can redirect the local flow across the caudal peduncle, but cannot affect the wake structures. The result is in agreement with the conclusion of Nauen and Lauder [14], though the morphology and kinematics of the finlets model are different from those of a real fish.

The performance of finlets could be sensitive to the size, position, morphology, and kinematics. Only rigid rectangular finlets undulating with the body have been studied 
in this work. To better understand the role of finlets, more complex models and motions of the finlets need to be investigated.

\section{Acknowledgments}

This work was supported by Chinese Academy of Sciences under the Innovative Project 'Multi-scale modeling and simulation in complex systems' (KJCX-SW-L08), 'Mathematical modeling of complex system' (KJCX3-SYW-S01), National Basic Research Program of China (973 Program) under Project No. 2007CB814800, and National Natural Science Foundation of China under Project Nos. 10702074 and 10872201. The computations were supported by the Supercomputing Center of Chinese Academy of Sciences and the Shanghai Supercomputer Center.

\section{References}

[1] R. Bainbridge, Training, speed and stamina in trout, J. Exp. Bio., 39 (1962), 537-555.

[2] T. Wu, Swimming of waving plate, J. Fluid Mech., 10 (1961), 321-344.

[3] M. Lighthill, Large amplitude elongated-body theory of fish locomotion, Proc. R. Soc. Lond. B, 179 (1971), 125-138.

[4] Q. Zhu, M. Wolfgang, and M. Triantafyllou, Three-dimensional flow structures and vorticity control in fish-like swimming, J. Fluid Mech., 468 (2002), 1-28.

[5] J. Zhang, S. Childress, A. Libchaber and M. Shelley, Flexible filaments in a flowing soap film as a model for one-dimensional flags in a two-dimensional wind, Nature, 408 (2000), 835-839.

[6] L. Zhu and C.S. Peskin, Simulation of a flapping flexible filament in a flowing soap film by the immersed boundary method, J. Comput. Phys. 179 (2002), 452-468.

[7] G. V. Lauder, P. Madden, R. Mittal, H. Dong and M. Bozkurttas, Locomotion with flexible propulsors I: experimental analysis of pectoral fin swimming in sunfish, Bioinsp. Biomim. 1 (2006), S25-S34.

[8] H. Dong, M. Bozkurttas, R. Mittal, P. Madden and G.V. Lauder, Computational modeling and analysis of the hydrodynamics of a highly deformable fish pectoral fin, J. Fluid Mech., 645 (2010), 345-373.

[9] P. Bandyopadhyay, Swimming and flying in nature-the route toward applications: the freeman scholar lecture, J. Fluids Eng., 131 (2009), 031801.

[10] J. Nauen and G. Lauder, Locomotion in scombrid fishes: morphology and kinematics of the finlets of the chub mackerel scomber japonicus, J. Exp. Biol., 203 (2000), 2247-2259.

[11] V. Walters, Body form and swimming performance in the scombroid fishes, Am. Zool., 2 (1962), 143-149.

[12] W. Webb, Hydrodynamics and energetic of fish propulsion, Bull. Fish. Res. Bd. Can., 190 (1975), 1-159.

[13] C. Lindsey, Form, function and locomotory habits in fish, in Fish Physiology volume VII: locomotion (edited by W.S. Hoar and D.J. Randall), Academic press, New York, 1978.

[14] J. Nauen and G. Lauder, Locomotion in scombrid fishes: visualization of flow around the caudal peduncle and finlets of the chub mackerel scomber japonicus, J. Exp. Biol., 204 (2001), 2251-2263. 
[15] C.J. Wu and L. Wang, Numerical simulation of self-propelled swimming of 3D bionic fish school, Sci. China Ser. E-Tec. Sci., 52 (2009), 658-669.

[16] C.S. Peskin, Flow patterns around heart valves: a numerical method, J. Comput. Phys., 10 (1972), 252-271.

[17] J. Yang and E. Balaras, An embedded-boundary formulation for large-eddy simulation of turbulent flows interacting with moving boundaries, J. Comput. Phys. 215 (2006), 12-40.

[18] N. Zhang and Z.C. Zheng, An improved direct-forcing immersed boundary method for finite difference applications, J. Comput. Phys. 221 (2007), 250-268.

[19] Z.G. Feng and E.E. Michaelides, The immersed boundary-lattice Boltzmann method for solving fluid-particles interaction problems, J. Comput. Phys. 195 (2004), 602-628.

[20] S.Z. Wang and X. Zhang, An immersed boundary method based on discrete stream function formulation for two- and three-dimensional incompressible flows, J. Comput. Phys. 230 (2011), 3479-3499.

[21] I. Borzjani and F. Sotiropoulos, Numerical investigation of the hydrodynamics of carangiform swimming in the transitional and inertial flow regimes, J. Exp. Biol., 211 (2008), 15411558.

[22] H. Dong, R. Mittal and F.M. Najjar, Wake topology and hydrodynamic performance of lowaspect-ratio flapping foils, J. Fluid Mech., 566 (2006), 309-343.

[23] K.D. Ellenrieder, K, Parker and J. Soria, Flow structures behind a heaving and pitching finitespan wing, J. Fluid Mech., 490 (2003), 129-138. 